\title{
Association of large intergenic noncoding RNA expression with disease activity and organ damage in systemic lupus erythematosus
}

Yanfang $\mathrm{Wu}^{1}$, Feifei Zhang ${ }^{2}$, Jianyang $\mathrm{Ma}^{2}$, Xiaoyan Zhang ${ }^{1}$, Lingling $\mathrm{Wu}^{1}, \mathrm{Bo} \mathrm{Qu}^{1}$, Shiwei Xia ${ }^{1}$, Shunle Chen ${ }^{1}$, Yuanjia Tang ${ }^{1 *}$ and Nan Shen ${ }^{1,2,3^{*}}$

\begin{abstract}
Introduction: Despite growing evidence that large intergenic noncoding RNAs (lincRNAs) can regulate gene expression and widely take part in normal physiological and disease conditions, our knowledge of systemic lupus erythematosus (SLE)-related lincRNAs remains limited. The aim of this study was to detect the levels of four lincRNAs (ENST00000500949: linc0949, ENST00000500597: linc0597, ENST00000501992: linc1992, and ENST00000523995: linc3995) involved in innate immunity in the peripheral blood mononuclear cells (PBMCs) of patients with SLE and correlate these lincRNA levels with disease activity, organ damage, clinical features and medical therapies.

Methods: PBMCs were obtained from 102 patients with SLE, 54 patients with rheumatoid arthritis (RA) and 76 healthy donors. lincRNA expression levels were measured by real-time quantitative polymerase chain reaction. Disease activity was assessed using the Systemic Lupus Erythematosus Disease Activity Index 2000 (SLEDAl-2K) scores, and organ damage was evaluated with the Systemic Lupus International Collaborating Clinics/American College of Rheumatology Damage Index.

Results: linc0949 and linc0597 were significantly decreased in patients with SLE compared with patients with RA and healthy control subjects. linc0949 was correlated with SLEDAI-2K score $(r=-0.329, P=0.0007)$, as well as with complement component C3 level $(r=0.348, P=0.0003)$. The level of linc0949 was also reduced in patients with SLE who had the presence of cumulative organ damage. In addition, decreasing expression of linc0949 was associated with lupus nephritis. linc0949 expression significantly increased after treatment, whereas neither disease activity nor organ damage correlated with linc0597 expression.
\end{abstract}

Conclusions: Our results provide novel empirical evidence that linc0949 could be a potential biomarker for diagnosis, disease activity and therapeutic response in SLE.

\section{Introduction}

Systemic lupus erythematosus (SLE) is a systemic autoimmune disease with various clinical manifestations affecting different tissues. It is characterized by the deposition of immune complexes due to widespread loss of immune tolerance to nuclear self-antigens, as well as by excessive proinflammatory cytokine production and damage to multiple organ systems [1]. Recent experimental and clinical

\footnotetext{
* Correspondence: tangyuanjia028@163.com; nanshensibs@gmail.com 'Shanghai Institute of Rheumatology, Department of Rheumatology, Renji Hospital, School of Medicine, Shanghai Jiao Tong University, Shan Dong Middle Road, Shanghai 200001, People's Republic of China

Full list of author information is available at the end of the article
}

studies have placed new emphasis on the role of the innate immune system in SLE. It has become apparent that Tolllike receptors (TLRs) can participate in cell activation by self molecules such as immune complexes containing DNA or RNA. Indeed, TLRs have an important role in the pathogenesis of lupus involving recruitment of adapter proteins; activation of protein kinases and transcription factors; and expression of inflammatory cytokines, chemokines, endothelial adhesion molecules and costimulatory molecules [2]. TLR signaling also stimulates B cell proliferation, cell differentiation and immunoglobulin class switching $[2,3]$. 
In the past, the importance of non-protein-coding RNAs has been emphasized in many biological and pathological processes [4]. Much research has been focused on microRNAs (miRNAs). miRNAs are small RNA molecules with a length of approximately 22 nucleotides (nt) that play a critical role in the pathogenesis of SLE by regulating gene expression at posttranscriptional levels $[5,6]$. miRNAs have also been reported to be involved in the local inflammatory response that ultimately leads to tissue injury and organ damage [7]. Recently, several studies have shown the feasibility of using miRNAs as biomarkers in body fluids for the diagnosis of SLE [8-10]. Though miRNAs play important roles in SLE, they are only a small fraction of the noncoding regions of the mammalian genome. Unlike miRNAs, long noncoding RNAs (lncRNAs) are expressed abundantly, including large intergenic noncoding RNAs (lincRNAs) [11].

lncRNAs are a class of mRNA-like transcripts ranging in length from $200 \mathrm{nt}$ to over $100 \mathrm{~kb}$ and lacking any significant open reading frames $[12,13]$. They are highly diverse and actively present in virtually every aspect of cell biology, such as cell differentiation, cell proliferation, DNA damage response, dosage compensation and chromosomal imprinting. Recently, a number of IncRNA molecules have been reported to be involved in diverse diseases [14-16]. Some evidence indicates that a few samples of lncRNAs could regulate the immune system [17-19]. In particular, there are several emerging hypotheses on IncRNA involvement in rheumatic diseases, such as rheumatoid arthritis (RA) [20,21], autoimmune thyroid disease [22] and psoriasis [23]. Other preliminary data in a murine model system pointed to a link between the lncRNA growth arrest-specific 5 (GAS5) and disease susceptibility to SLE [16]. In addition, the chromosomal locus of GAS5, 1q25, was showed to be associated with human SLE development in genetic studies [23-25].

Because of the heterogeneous presentation of patients with SLE and their unpredictable disease course, there is a pressing need to identify biomarkers that will facilitate better diagnosis and prognosis, and lincRNAs as biomarkers are still largely unexplored in this regard. It has been reported that four lincRNAs (linc0949, linc0597, linc1992 and linc3995) not only are differentially expressed following innate activation of THP-1 macrophages but also regulate induction of proinflammatory cytokines such as tumor necrosis factor (TNF)- $\alpha$ and interleukin (IL)-6 [26]. Moreover, it is well-established that IL-6 and TNF- $\alpha$ are involved in SLE pathogenesis [27-29].

As mentioned above, we hypothesized that these lincRNAs would produce cross-linking with SLE via innate immunity and play a critical role in the pathogenesis of SLE and that they might serve as biomarkers of disease activity, organ damage and medical response. In the present study, we aimed to investigate whether the expression levels of these lincRNAs in peripheral blood mononuclear cells (PBMCs) were abnormal in patients with SLE, assess the relationship of the levels with disease activity and organ damage, and explore new biomarkers used in disease monitoring and prognostication.

\section{Methods}

\section{Patients and healthy controls}

All samples from patients with SLE and patients with RA were obtained from the Department of Rheumatology of Renji Hospital (Shanghai, China). All patients with SLE met at least four of the American College of Rheumatology (ACR) 1982 revised criteria for SLE [30]. Patients with RA were diagnosed according to the ACR/European League Against Rheumatism 2010 classification criteria for RA [31]. The control group comprised healthy volunteers with no history of autoimmune disease or immunosuppressive therapy. Otherwise eligible individuals with a current or recent infection were excluded from the study. Control subjects were frequency-matched with the patients for age and sex. All participants were from the Han Chinese population. The study was approved by the Research Ethics Board of Renji Hospital, Shanghai Jiao Tong University School of Medicine, Shanghai, China. Informed consent was obtained from all study participants.

The patients with lupus were all receiving steroid therapy at the time of the study, and a prednisone dosage per day (dosages of other steroids were converted to prednisone equivalents) from $2.5 \mathrm{mg}$ to $500 \mathrm{mg}$ (mean dosage: $29.5 \mathrm{mg} /$ day). In addition, 42 patients were receiving immunosuppressive therapy (azathioprine (AZA; $n=7$ ), cyclophosphamide (CYC; $n=10)$, cyclosporine $A(C s A ; n=6)$, tacrolimus (FK506; $n=1$ ), leflunomide (LEF; $n=2$ ), mycophenolate mofetil (MMF; $\mathrm{n}=8$ ), methotrexate (MTX; $\mathrm{n}=$ 8)), and 59 were receiving an antimalarial drug (chloroquine or hydrochloroquine). For each patient, the severity of disease was assessed with the Systemic Lupus Erythematosus Disease Activity Index 2000 (SLEDAI-2K) [32]. Organ damage (defined as nonreversible change, not related to active inflammation, occurring since the onset of lupus and present for at least 6 months) was assessed using the Systemic Lupus International Collaborating Clinics/American College of Rheumatology Damage Index (SDI) score [33]. In our cohort, nearly $51.0 \%$ of patients (52 of 102 patients with SLE) had either previous or current lupus nephritis (LN) (Table 1). Subjects were considered to have active renal disease if proteinuria was $\geq 0.5 \mathrm{~g} /$ day, hematuria was $\geq 5$ red blood cells per high-power field (hpf), pyuria was $\geq 5$ white blood cells/hpf or cellular casts were present. Infection, kidney stones and other causes were excluded.

\section{Peripheral blood samples handling and RNA processing}

Peripheral blood samples $(10 \mathrm{ml})$ were obtained from each subject. The samples were collected in tubes containing 
Table 1 Large intergenic noncoding RNA linc0949 by presence or absence of clinical features of systemic lupus erythematosus $^{a}$

\begin{tabular}{|c|c|c|c|c|c|}
\hline \multirow[t]{2}{*}{ Clinical features } & \multicolumn{2}{|c|}{ SLE clinical features present } & \multicolumn{2}{|c|}{ SLE clinical features absent } & \multirow[t]{2}{*}{$P$-value } \\
\hline & $\mathrm{N}$ & Mean \pm SEM (range, $10^{-3}$ ) & $\mathrm{N}$ & Mean \pm SEM $\left(\right.$ range, $10^{-3}$ ) & \\
\hline Renal & 50 & $2.16 \pm 0.152$ & 52 & $1.47 \pm 0.132$ & 0.0014 \\
\hline Rash & 33 & $1.89 \pm 0.200$ & 69 & $1.87 \pm 0.199$ & NS \\
\hline Arthritis & 35 & $1.79 \pm 0.327$ & 67 & $1.97 \pm 0.163$ & NS \\
\hline Serositis & 21 & $1.81 \pm 0.307$ & 81 & $1.72 \pm 0.364$ & NS \\
\hline Mucosal ulcer & 19 & $2.02 \pm 0.207$ & 83 & $1.87 \pm 0.187$ & NS \\
\hline Hematologic & 30 & $1.84 \pm 0.243$ & 72 & $2.06 \pm 0.179$ & NS \\
\hline Neurologic & 9 & $1.99 \pm 0.084$ & 93 & $2.05 \pm 0.45$ & NS \\
\hline \multicolumn{6}{|l|}{ Autoantibodies } \\
\hline Anti-dsDNA & 40 & $1.91 \pm 0.274$ & 62 & $1.88 \pm 0.264$ & NS \\
\hline Anti-Sm & 16 & $2.07 \pm 0.202$ & 86 & $1.78 \pm 0.193$ & NS \\
\hline Anti-nucleosome & 40 & $1.91 \pm 0.204$ & 62 & $1.88 \pm 0.212$ & NS \\
\hline Anti-SSA/SSB & 32 & $2.03 \pm 0.258$ & 70 & $2.01 \pm 0.176$ & NS \\
\hline Anti-RNP & 22 & $1.95 \pm 0.253$ & 80 & $1.96 \pm 0.165$ & NS \\
\hline \multicolumn{6}{|l|}{ Medical therapy } \\
\hline Prednisone dose $\geq 30 \mathrm{mg} /$ day & 48 & $2.08 \pm 0.175$ & 54 & $1.94 \pm 0.181$ & NS \\
\hline Immunosuppressants ${ }^{\mathrm{b}}$ & 42 & $2.00 \pm 0.141$ & 60 & $1.54 \pm 0.151$ & 0.0365 \\
\hline
\end{tabular}

${ }^{a}$ dsDNA, Double-stranded DNA; NS, Not significant; RNP, Ribonucleoprotein; SEM, Standard error of the mean; SLE, Systemic lupus erythematosus; Sm, Smith; SSA, Sjögren's syndrome-related antigen A; SSB, Sjögren's syndrome-related antigen B. 'Immunosuppressants included azathioprine, cyclophosphamide, cyclosporine A, FK506 (tacrolimus), leflunomide, mycophenolate mofetil and methotrexate.

ethylenediaminetetraacetic acid (EDTA). PBMCs were isolated from anticoagulated whole blood by use of Ficoll density gradient centrifugation. Then total RNA was extracted from PBMCs using TRIzol reagent (Invitrogen, Carlsbad, CA, USA). The integrity of the RNA was assessed using capillary gel electrophoresis, and the concentrations of RNA were measured using a NanoDrop $^{\text {тM }} 1000$ spectrophotometer (NanoDrop Technologies, Wilmington, DE, USA) with a $260 \mathrm{~nm} / 280 \mathrm{~nm}$ ratio above 1.8. About $200 \mathrm{ng}$ of total RNA were reverse-transcribed into cDNA using a PrimeScript RT reagent kit (Takara Bio, Dalian, China). All RNA and cDNA samples were stored at $-70^{\circ} \mathrm{C}$ before use.

\section{Cell culture and stimulation}

Peripheral blood samples were obtained from five healthy donors and five patients with SLE. The samples were collected in tubes containing EDTA. PBMCs were isolated from anticoagulated whole blood by Ficoll density gradient centrifugation. Two hours before stimulation, $1 \times 10^{6}$ PBMCs were cultured in 24-well flat-bottomed plates in $500 \mu \mathrm{l}$ of RPMI 1640 medium containing $10 \%$ fetal bovine serum (FBS). Then the PBMCs were stimulated for 4 hours with the TLR2 ligand Pam3CK4 (20 ng/ml).
Cell culture and treatment with dexamethasone and immunosuppressant agents

Peripheral blood samples were obtained from two healthy donors. PBMCs were isolated from anticoagulated whole blood by use of Ficoll density gradient centrifugation. PBMCs $\left(1 \times 10^{6}\right)$ were resuspended for 2 hours in $500 \mu \mathrm{l}$ of RPMI 1640 medium containing 10\% FBS, then dexamethasone was added with the indicated concentration (Dexamethasone concentrations were $10 \mathrm{ng} / \mathrm{ml}, 100 \mathrm{ng} / \mathrm{ml}, 1000 \mathrm{ng} / \mathrm{ml}, 10 \mathrm{ug} / \mathrm{ml}$, respectively) for another 24 hours, as were CsA (200 nmol) and FK506 (20 nmol). RNA samples were then isolated, and real-time quantitative PCRs (RT-qPCRs) were performed.

\section{Real-time quantitative polymerase chain reaction}

To quantify the expression of four lincRNAs (linc0949, linc0597, linc1992 and linc3995), cDNA was amplified by RT-PCR with SYBR Green (SYBR Premix Ex Taq RT-PCR kit; Takara Bio). The primer sequences used for SYBR Green-based RT-PCR are given in Table 2. The ribosomal protein L13A (RPL13A) gene was used as an internal control to normalize the amounts of cDNA. The SYBR Green assays were performed in duplicate using an ABI ViiA 7 Real-Time PCR System (Applied Biosystems, Foster City, CA, USA). The relative expression levels were calculated using the $2^{-\Delta C t}$ comparative threshold cycle method. 
Table 2 Primers used to amplify transcripts of large intergenic noncoding RNAs ${ }^{a}$

\begin{tabular}{lll}
\hline Gene & Forward & Reverse \\
\hline RPL13A & 5'-CTGGAGGAGAAGAGGAAAGAGA-3' & 5'-TTGAGGACCTCTGTGTATTTGTCAA-3' \\
ENST00000500597 & 5'-TTGGATTCATCCCGTTCACCTCCA-3' & 5'-CAGCATGACGATCAAGCGAGATTC-3' \\
ENST00000501992 & 5'-AACTCCTGACCTCAGGTGATCCAT-3' & 5'-AAGGGAGTTCAGAAGGTGTGGCT-3' \\
ENST00000500949 & 5'-TCCTGCAACCCAAGGTGGATACTT-3' & 5'-CTGCAGTGAGCAGAAATCACGCAT-3' \\
ENST00000523995 & 5'-GTTGTGGCATATGGCTCTGCTGT-3' & 5'-CATTGCAGGAAAGAGTGCCAAGGT-3' \\
\hline
\end{tabular}

${ }^{\text {a }}$ The four large intergenic noncoding RNAs primers are derived from the literature [26].

\section{Statistical analysis}

Data were analyzed with GraphPad Prism version 5.0 software (GraphPad Software, La Jolla, CA, USA). The nonparametric Mann-Whitney $U$ test was used to compare gene expression between two groups. The correlation between groups was evaluated using Spearman's rank correlation coefficient test. The strength of the correlation was graded using Cohen's criteria as follows: 0.3 to $0.5=$ weak, 0.5 to $0.7=$ moderate and $>0.7=$ strong [34]. P-values (two-tailed) $<0.05$ were considered statistically significant.

\section{Results}

Decreased linc0949 and linc0597 levels in patients with systemic lupus erythematosus

The expression levels of four lincRNAs (linc0949, linc0597, linc1992 and linc3995) in PBMCs taken from 102 patients with SLE, 54 patients with RA and 76 healthy donors were measured using RT-qPCR. Patients with SLE and healthy donors did not differ significantly with respect to mean age or sex distribution (Table 3). The average disease duration of patients enrolled in our study was 4.98 years for those with SLE and 5.12 years for those with RA. In general, the patients with SLE had mild to moderate flares of disease activity and severity, with a mean SLEDAI-2K score of 7 and a mean SDI of 0.78 (Table 3).

As shown in Figure 1A, patients with SLE had significantly lower linc0949 levels than healthy donors and patients with RA (both $P<0.0001$ ). Also, the expression of linc0597 was decreased dramatically in patients with
SLE compared with healthy donors and patients with RA $(P=0.0001$ and $P<0.0001$, respectively) (Figure $1 \mathrm{~B}$ ). Figure $1 \mathrm{C}$ and Figure 1D, however, show no significant differences in linc1992 and linc3995 levels between patients with SLE and healthy donors or patients with RA. These results revealed that lower expression of linc0949 and linc0597 was specific to SLE, so we selected these lincRNAs for further research.

\section{Association of linc0949 level with disease activity in patients with systemic lupus erythematosus}

To investigate whether the expression of linc0949 and linc0597 is related to SLE disease activity, we compared the relative expression levels of the lincRNAs in patients with SLE with different levels of disease activity, assessed on the basis of SLEDAI-2K score and the level of complement C3. In accordance with the SLEDAI-2K flare system, patients with SLE were divided into those with stable disease (SLEDAI-2K scores from 0 to 4 ), those with a mild flare (SLEDAI-2K scores from 5 to 10) and those with a moderate to severe disease flare (SLEDAI$2 \mathrm{~K}$ scores $>10$ ). linc0949 was significantly lower in patients with SLE who had a mild flare or a moderate to severe flare of disease than in patients without a flare $(P=0.0032$ and $P=0.0004$, respectively) (Figure $2 \mathrm{~A}$ ). In addition, a correlation between linc0949 and SLEDAI-2K score was observed in that decreased levels of linc0949 coincided with increased SLEDAI-2K score in patients with SLE $(r=-0.329, P=0.0007)$ (Figure 2B).

Table 3 Demographic data ${ }^{a}$

\begin{tabular}{|c|c|c|c|}
\hline & Patients with SLE $(n=102)$ & Patients with RA $(n=54)$ & Healthy donors $(n=76)$ \\
\hline Age (yr) & $34.3 \pm 1.3(16$ to 65$)$ & $38.7 \pm 2.3(20$ to 65$)$ & $34.0 \pm 1.2(20$ to 63$)$ \\
\hline \multicolumn{4}{|l|}{$\operatorname{Sex}(n)$} \\
\hline Female & 93 & 46 & 70 \\
\hline Male & 9 & 8 & 6 \\
\hline Disease duration (yr) & $4.98 \pm 0.76$ (0.04 to 28$)$ & $5.12 \pm 0.84(0.5$ to 20$)$ & - \\
\hline ANA (\%) & 95.0 & - & - \\
\hline SLEDAI-2K & $6.82 \pm 0.52(1$ to 15$)$ & - & - \\
\hline SDI & $0.78 \pm 0.14(0$ to 3$)$ & - & - \\
\hline
\end{tabular}

${ }^{a}$ ANA, antinuclear antibody; RA, Rheumatoid arthritis; SDI, Systemic Lupus International Collaborating Clinics/American College of Rheumatology Damage Index SLE, Systemic lupus erythematosus; SLEDAI-2K, Systemic Lupus Erythematosus Disease Activity Index 2000 score. 

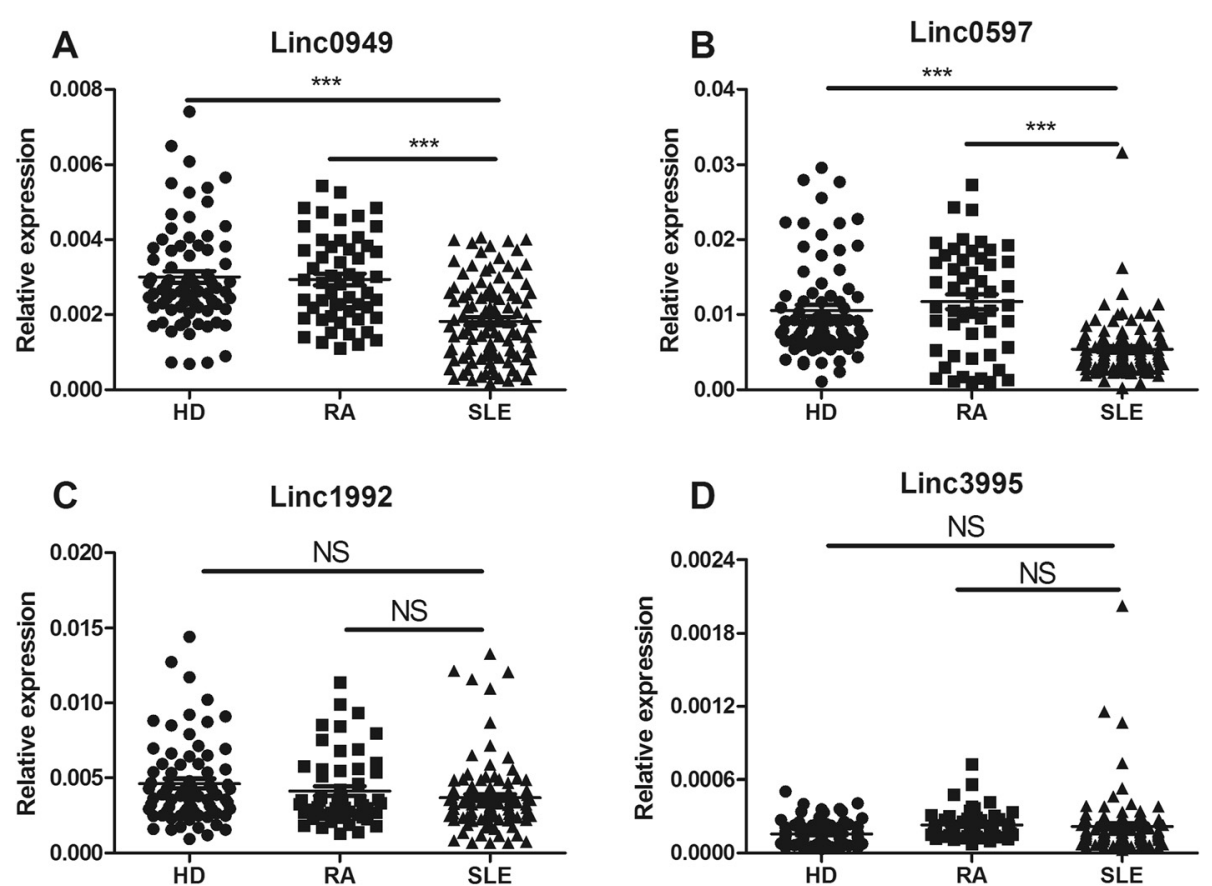

Figure 1 Comparison of expression of four large intergenic noncoding RNAs (linc0949, linc0597, linc1992 and linc3995) between patients with SLE, patients with RA and healthy donors. Each symbol represents an individual patient; horizontal lines indicate median values. The expression levels of the four large intergenic noncoding RNAs (lincRNAs) in 102 patients with SLE, 54 patients with RA and 76 healthy donors were analyzed by real-time quantitative PCR and normalized by RPL13A level. (A) Decreased expression of linc0949 in patients with SLE versus patients with RA and healthy donors. (B) linc0597 expression was significantly reduced in patients with SLE versus patients with RA and healthy donors. No apparent difference was detected in the expression of linc1992 (C) and linc3995 (D) between patients with SLE, patients with RA and healthy donors. NS, Not significant. ${ }^{* * *} P<0.001$. HD, Healthy donors; RA, Rheumatoid arthritis; SLE, Systemic lupus erythematosus.

C3 level is also an indicator of disease activity. Hypocomplementemia is often observed in patients with SLE with active disease. In the present research, linc0949 expression was significantly decreased in patients with SLE who had a reduced level of complement C3 $(<80 \mathrm{mg} / \mathrm{dl})$ compared with those with normal levels of $\mathrm{C} 3(P<0.0001)$ (Figure $2 \mathrm{C}$ ). Further analysis revealed a positive correlation between linc0949 level and C3 level $(r=0.348, P=$ 0.0003) (Figure 2D). However, the level of linc0597 did not correlate with SLEDAI-2K and complement C3 level (data not shown). These results indicate that the abnormal expression of lincRNA0949 may be a key indicator of disease activity in patients with SLE.

\section{Reduced expression of linc0949 in patients with systemic} lupus erythematosus with organ damage

SLE is a chronic multisystem autoimmune disease that can affect virtually every organ system and may lead to significant morbidities. Assuming that lincRNAs are involved in tissue damage and inflammation, we investigated whether linc0949 and linc0597 were associated with different levels of chronic and irreversible organ damage in the patients with SLE. The results revealed significantly lower levels of linc0949 in patients with SLE with SDI scores of 1 to 2 and in those with scores $>2$ versus those without organ damage $(P=0.0059$ and $P=0.0009$, respectively) (Figure 3A). linc0597 expression was not significantly reduced in patients with organ damage (SDI $\geq 1$ ) versus those who remained damage-free (data not shown).

$\mathrm{LN}$ is one of the most common clinical manifestations and causes of organ damage in patients with SLE, so we wanted to know whether lincRNA levels are related to LN. In our cohort, nearly $51 \%$ of patients (52 of 102 patients with SLE) had either previous or current LN (Table 1). Patients with LN had lower linc0949 expression levels than those without renal manifestations $(P=$ 0.0014) (Figure 3B). Expression levels of linc0949 were decreased in the group of patients with active LN compared with those without LN $(P=0.0009)$ (Figure 3C), whereas linc0949 levels were not significantly different in patients with inactive LN compared with patients without $\mathrm{LN}$ at the time of blood drawing $(P=0.0739)$ (Figure 3C). These data suggest that linc0949 expression is related with cumulative organ damage in SLE and that linc0949 may be useful in predicting long-term outcome and prognosis in patients with SLE. 

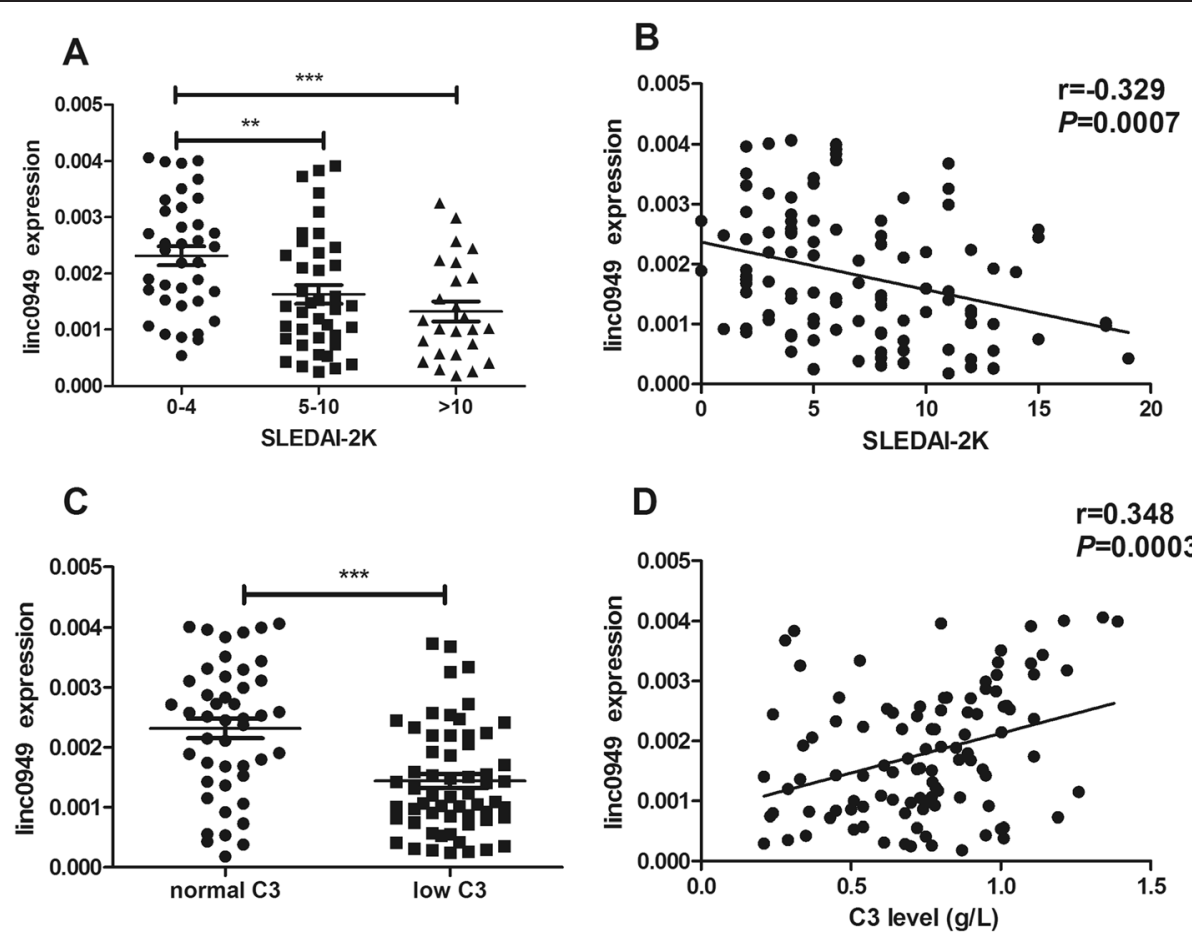

Figure 2 Association of large intergenic noncoding RNA linc0949 expression with disease activity in patients with systemic lupus erythematosus. (A) Patients with systemic lupus erythematosus (SLE) with a moderate to severe flare of disease (Systemic Lupus Erythematosus Disease Activity Index 2000 (SLEDAl-2K) score >10) or a mild flare of disease (SLEDAI-2K score from 5 to 10) had significantly lower large intergenic noncoding RNA linc0949 expression than did those without a disease flare (SLEDAI-2K score <4) at the time of blood donation. (B) linc0949 expression was negatively correlated with SLEDAI-2K score. (C) linc0949 expression was significantly decreased in patients with SLE with a reduced level of complement C3 (<80 mg/dl) compared with those with normal C3levels. (D) A significantly positive correlation was observed between linc0949 expression and C3 level in patients with SLE. ${ }^{* *} P<0.01,{ }^{* *} P<0.001$.

Relationship of large intergenic noncoding RNA levels with clinical manifestations and medical therapies

To assess the association between lincRNA levels and clinical manifestations, autoantibody profiles and medical treatments, linc0949 and linc0597 levels were compared between patients with certain clinical features and those without certain clinical features. We identified no association between linc0949 or linc0597 expression and clinical manifestations such as rash, arthritis, serositis, mucosal ulcer, hematologic involvement or neurologic manifestations (linc0949: Table 1; linc0597: data not shown). We also found that neither linc0949 nor linc0597 appeared
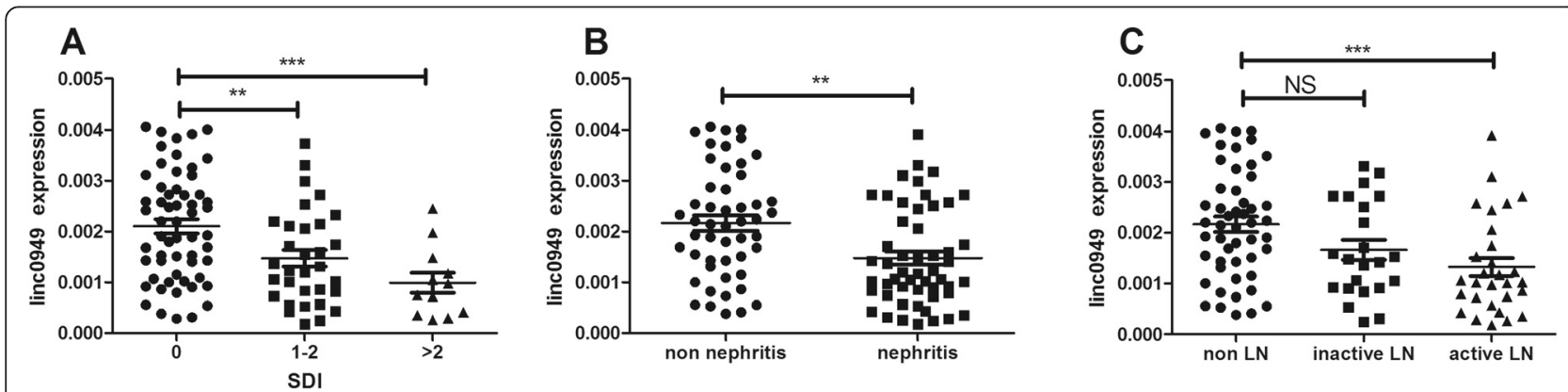

Figure 3 Large intergenic noncoding RNA linc0949 expression was decreased in patients with systemic lupus erythematosus who had organ damage. (A) In our cohort, linc0949 was dramatically decreased in patients with systemic lupus erythematosus (SLE) who had chronic and irreversible organ damage (Systemic Lupus International Collaborating Clinics/American College of Rheumatology Damage Index (SDI) scores 1 to 2 or more) compared with those with no damage. (B) Large intergenic noncoding RNA linc0949 expression exhibited a decreasing trend in patients with lupus nephritis ( $L N ; n=52)$ relative to patients with no history of $L N(n=50)$. (C) Patients with active $L N$ had lower linc0949 expression than those without renal manifestations, whereas linc0949 level was not significantly different in patients with inactive LN compared with those without LN. NS, Not significant. ${ }^{* *} P<0.01,{ }^{* * *} P<0.001$. 
to be associated with autoantibody production, including anti-double-stranded DNA, anti-Smith antibodies, antinucleosome antibodies, anti-Sjögren's syndromerelated antigen A and B antibodies and antiribonucleoprotein antibodies (linc0949: Table 1; linc0597: data not shown).

When medical therapies were considered, the expression of linc0949 exhibited no significant difference in patients receiving medium to high doses of prednisone (>30 mg/day) compared with patients treated with low doses of prednisone (Figure 4A). By contrast, the expression of linc0949 in patients with SLE being treated with immunosuppressants (AZA, CYC, CsA, LEF, MMF, MTX and FK506) was significantly reduced compared with those not receiving immunosuppressants at the time of blood donation $(P=0.0365)$ (Figure $4 B$ ).

We next sought to assess whether SLE disease activity or antirheumatic drugs affect the expression of linc0949, as it was found that some lncRNAs can be induced in response to the anti-inflammatory agent dexamethasone [35]. Thus, we treated PBMCs of two healthy donors with different concentrations of dexamethasone, CsA and FK506. FoxP3 level was significantly reduced after treatment (Additional file 1: Figure S1), which supported the fact that dexamethasone and immunosuppressive agents worked effectively in vitro. As shown in Figure 4C, dexamethasone, CsA and FK506 did not affect the expression of linc0949. This result relates to the effects of antirheumatic drugs on the expression of linc 949 , which confirms that linc0949 is intrinsically underexpressed in patients with SLE.

We next investigated whether linc0949 was responsive to treatment and changes over time in conjunction with disease activity. We chose three patients with SLE(P1,P2,P3), as described in Figure 4D, P1 and P3 had initial onset of biopsy-proved type IV LN, P2 had neuropsychological lupus, and their peripheral blood samples were collected at both the beginning of treatment and after 12 weeks of treatment. P1 and P3 used high-dose prednisone $(1 \mathrm{mg} / \mathrm{kg}$ per day) plus mycophenolate mofetil (1.5-2 g/day), whereas P2 used repeated pulses of glucocorticoid (500 mg intravenous methylprednisolone per day) for three days and then prednisone ( $1 \mathrm{mg} / \mathrm{kg}$ per day) plus monthly pulse of cyclophosphamide $(0.8 \mathrm{~g} / \mathrm{month})$. After treatment, all of
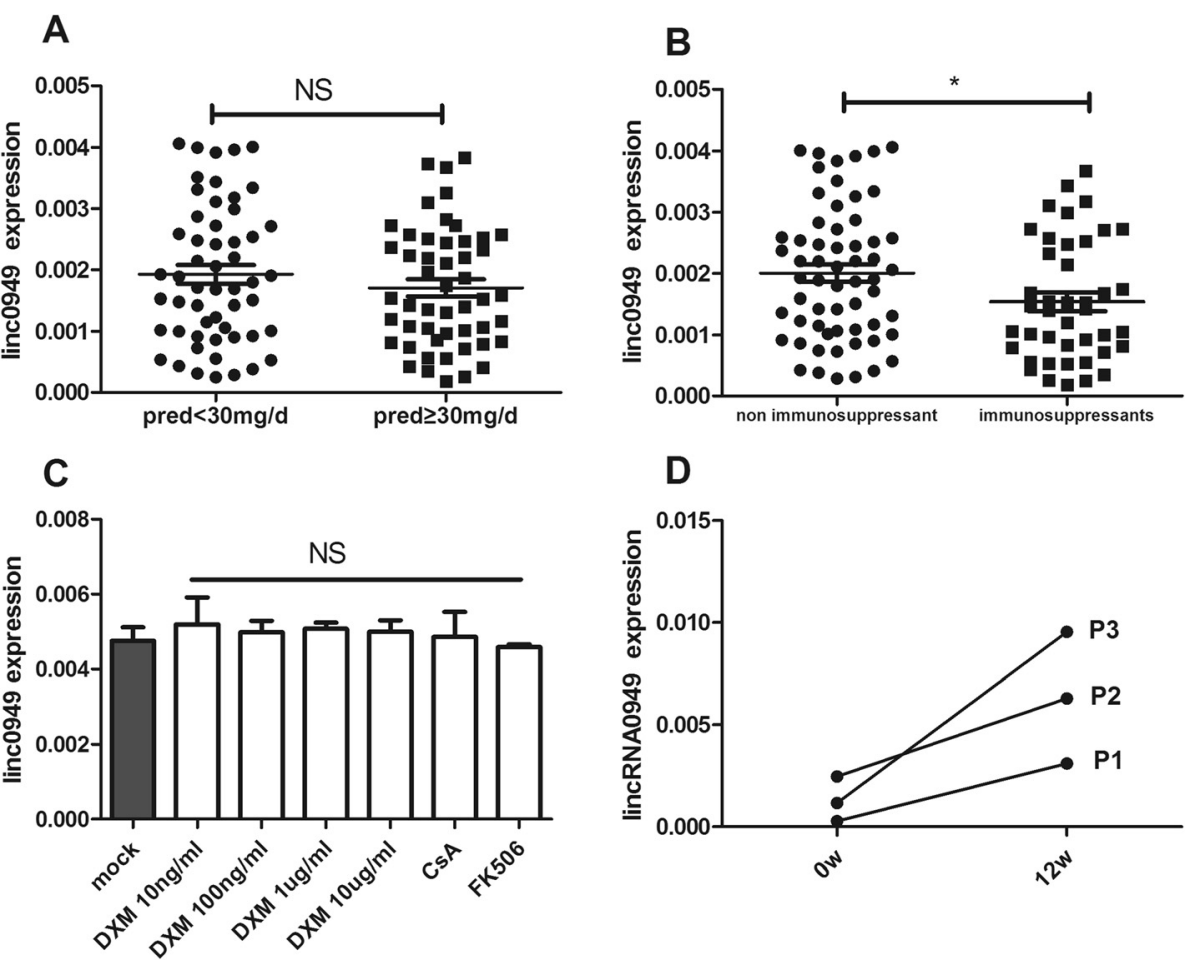

D

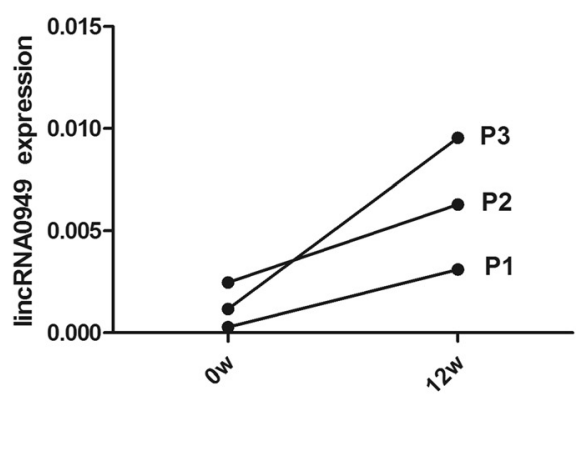

Figure 4 Association of large intergenic noncoding RNA linc0949 level with medical therapies in patients with systemic lupus erythematosus. (A) The level of the large intergenic noncoding RNA linc0949 had no apparent difference in patients with systemic lupus erythematosus (SLE) whose daily dosage of prednisone was $>30 \mathrm{mg}$ compared with those whose daily dosage of prednisone was <30 mg. (B) linc0949 expression decreased significantly in patients with SLE who had used immunosuppressants (azathioprine, cyclophosphamide, cyclosporine A (CSA), leflunomide, mycophenolate mofetil, methotrexate, FK506) compared with those without immunosuppressant treatment. (C) The expression of linc0949 was not related to the use of drugs. The data are from two healthy donors. (D) linc0949 expression was detected in three patients with active SLE at the beginning of and after 12 weeks of treatment. As we observed, the linc0949 expression of all three patients was notably increased when they achieved significant clinical improvement after treatment. ${ }^{*} P<0.05$. DXM, Dexamethasone; NS, Not significant; Pred, Prednisone. 
the three patients achieved clinical remission, with the urinary protein level dropping to less than $0.5 \mathrm{~g} / 24$ hour in P1 and P3; and with cerebrospinal fluid inspection and head MRI restoring to normal in P2. SLEDAI-2K score of the three patients reduced to a stable level (Additional file 1: Figure S1). In concordance with the clinical improvement, linc0949 expression in these three patients also significantly increased (Figure 4D).

\section{Abnormal regulation of lincRNAs during innate activation of peripheral blood mononuclear cells in patients with systemic lupus erythematosus}

To investigate the different response to innate immunity of linc0949 and linc0597 in healthy donors and patients with SLE, we stimulated PBMCs from healthy donors and patients with SLE with TLR2 ligands. We chose TLR2 ligands because it was reported that linc0949 and linc0597 were regulated in THP-1 macrophages following Pam3CSK4 stimulation [26] and TLR2 was required for the production of prototypical lupus autoantibodies and the development of renal disease in murine lupus [36,37]. To prepare cell samples, PBMCs obtained from 5 healthy donors and 5 patients with SLE were stimulated with Pam3CSK4 for 4 hours. Then we performed RT-qPCR to identify changes in lincRNA expression. In PBMCs of healthy controls, linc0949 was suppressed (Figure 5A), while linc0597 was increased (Figure 5B) after treatment with Pam3CSK4. But in patients with SLE, linc0949 and linc0597 could not response to the stimuli compared with healthy donors (Figure 5A and B). These results demonstrated that lincRNAs were indeed involved in the complex regulatory network of innate immunity.

\section{Discussion}

In recent years, an increasing body of evidence has shown that lncRNAs play major biological roles in embryogenesis, stem cell biology and cellular development and show developmental and tissue-specific expression patterns $[11,38,39]$. Studies have also suggested that abnormal expression of lncRNAs might be associated with numerous diseases, indicating that these RNAs may open a new avenue for diagnostic and therapeutic targets by recognition of their roles in human disease.

In the present study, we detected four lincRNAs (linc0949, linc0597, linc1992 and linc3995) and investigated the association between their expression levels and specific clinical features of SLE. Two of these lincRNAs (linc0949 and linc0597) were significantly decreased in patients with SLE compared with healthy donors and disease controls. linc0949 was associated with disease activity, as assessed using the SLEDAI-2K score and C3 level in patients with SLE. Moreover, linc0949 expression was reduced in patients with SLE with ongoing or cumulative organ damage, as assessed based on SDI score or the presence of active LN. linc0949 expression does not participate in clinical manifestations other than LN, which demonstrates that it has very good detection specificity for LN. Lower levels of linc0949 may thus be helpful to identify patients with SLE who have active and severe disease. To evaluate the effect of antirheumatic drugs on

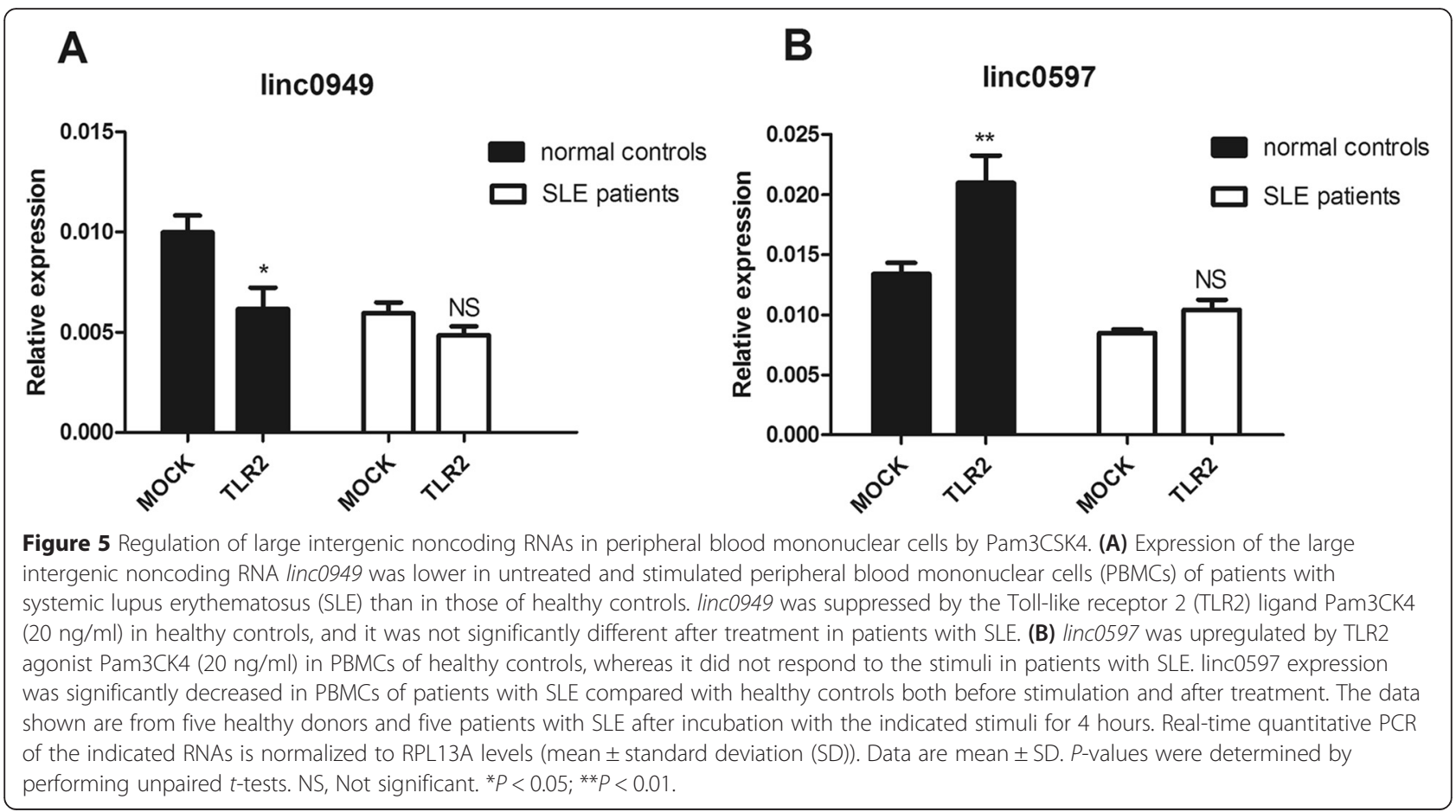


the expression of linc 949 , we used in vitro studies to test whether the addition of dexamethasone, CsA or FK506 to cultured PBMCs would affect the expression of linc0949. As shown in Figure 4C, these antirheumatic drugs did not affect the expression of linc0949 in PBMCs, which confirmed that linc0949 was intrinsically underexpressed in patients with SLE. linc0949 expression of three patients with severe disease flares significantly increased after treatment (Figure 4D), indicating that linc0949 might be responsive to treatment and might change in conjunction with disease activity and severity and suggesting that linc0949 might be used to monitor disease progression and guide therapy.

Over the past several decades, tremendous enthusiasm and efforts have been devoted to biomarkers for SLE because the diagnosis of SLE requires a combination of clinical manifestations and biomarkers and no single test is sufficiently sensitive and specific to be diagnostic. The traditional antibodies fail to identify the pathogenic processes, organ damage and biological responses to a therapeutic intervention. Many groups, including the members of our laboratory, have found a set of potential biomarkers for SLE. For example, interferon (IFN)-induced genes and IFN-inducible chemokines may serve as new biomarkers for active and severe disease in patients with SLE [40,41]. Some limitations of these biomarkers are revealed gradually, however. Several studies have shown that overexpressed transcripts of the type I IFN pathway are also identified in patients with myositis, RA, Sjögren's syndrome and scleroderma [42-44], so an IFN signature or chemokine is not sufficiently specific. In two longitudinal studies to date, researchers have reported conflicting results on the correlations between type I IFN gene signature score and diseases activity $[45,46]$. There is an urgent need for SLE biomarkers that can help enhance comprehension of the mechanisms of diseases or effects of therapies by relating the changes of molecular and cellular pathways to disease status or clinical responses. In our present study, we demonstrate that lower expression of linc0949 is specific for SLE and that it is helpful in identifying disease activity, monitoring disease progression and guiding therapy. However, linc0949 needs to be further investigated in large-scale multicenter trials.

On the basis of our present observations, we believe that linc0949 could be a potentially readily accessible biomarker useful for diagnosing SLE. As a novel biomarker, lincRNAs have the following characteristics. First, lincRNAs display a wide range of stabilities in the samples comparable to those of mRNAs of protein-coding genes [47]. Second, they show a greater tissue specificity compared with protein-coding mRNAs and miRNAs, which are frequently expressed in multiple tissues, and they show highly increased or decreased expression levels in disease [15]. In addition, lincRNAs are also detectible in body fluids such as plasma and urine [48-50], diagnostic samples of which are easy to collect using noninvasive methods. Moreover, detection of the lincRNAs is simple, inexpensive and has high throughput, making it a suitable approach to gaining an overview of disease activity and severity in patients with SLE. These features make lincRNAs very suitable as biomarkers, and many studies have been published on this matter in recent years, both in cancer and in other human diseases such as cardiovascular diseases [50] and neurological disorders [51].

Most lncRNAs described to date have been found to be related to transcriptional regulation or mRNA processing, characteristics that they share with microRNAs. However, unlike microRNAs, IncRNAs show a greater complexity of their functions and have a wider spectrum of biological contexts, such as epigenetic regulation, enhancer-like function and RNA splicing, editing and export [52]. In our ongoing experiments, we found that linc0949 and linc0597 could be induced by TLR2 in PBMCs of healthy donors, but they did not respond to the stimuli in patients with SLE as compared with healthy donors (Figure 5). These results validate that lincRNAs were indeed involved in the complex regulatory network of innate immunity. We hypothesized that the regulation defect of linc0949 and linc0597 could contribute to the pathogenesis of SLE and that lincRNAs may provide potential novel strategies for therapeutic intervention, although their function and mechanism of action need further exploration.

We have suggested the abnormal expression of linc0949 in patients with SLE, as well as the association of lincRNA level with disease activity and organ damage; however, in this study, we did not conduct a functional study of this lincRNA, and the underlying mechanism needs further investigation. We did not detect the expression of linc0949 released in the local target tissues and in specific cell subsets in PBMCs. Future studies are needed to investigate the lincRNA expression level in specific organ and cell types as well.

\section{Conclusions}

We found that the expression of two lincRNAs was dramatically reduced in patients with SLE and that the decreasing level of linc0949 was correlated with disease activity, degree of organ damage and medical therapies in patients with SLE. linc0949 may serve as a potential biomarker for diagnosis, disease activity and therapeutic interventions in patients with SLE.

\section{Additional file}

Additional file 1: Figure S1. Effectiveness of the amplified drugs and SLEDAI score flare of three patients. 


\section{Abbreviations}

ACR: American College of Rheumatology; ANA: Antinuclear antibody; AZA: Azathioprine; C3: Complement component 3; CsA: Cyclosporine A; CYC: Cyclophosphamide; dsDNA: Double-stranded DNA; DXM: Dexamethasone; EDTA: Ethylenediaminetetraacetic acid; FBS: Fetal bovine serum; FK506: Tacrolimus; GAS5: Growth arrest-specific 5; hpf: High-power field; IFN: Interferon; IL: Interleukin; LEF: Leflunomide; linc0597: ENST00000500597; linc0949: ENST00000500949; linc1992: ENST00000501992;

linc3995: ENST00000523995; lincRNA: Large intergenic noncoding RNA; LN: Lupus nephritis; IncRNA: Long noncoding RNA; miRNA: microRNA MMF: Mycophenolate mofetil; MTX: Methotrexate; NS: Not significant; nt: Nucleotide; PBMC: Peripheral blood mononuclear cell; RA: Rheumatoid arthritis; RNP: Ribonucleoprotein; RPL13A: Ribosomal protein L13A gene; RT-qPCR: Real-time quantitative polymerase chain reaction; SDI: Systemic Lupus International Collaborating Clinics/American College of Rheumatology Damage Index; SEM: Standard error of the mean; SLE: Systemic lupus erythematosus; SLEDAI-2K: Systemic Lupus Erythematosus Disease Activity Index 2000; SLICC: Systemic Lupus International Collaborating Clinics; Sm: Smith; SSA: Sjögren's syndrome-related antigen A; SSB: Sjögren's syndrome-related antigen B; TLR: Toll-like receptor; TNF: Tumor necrosis factor; SEM: Standard error of the mean.

\section{Competing interests}

The authors declare that they have no competing interests.

\section{Authors' contributions}

YFW, YJT and NS conceived of and designed the experiments and analyzed the data. YFW, FFZ, YJT and NS wrote the paper. YFW, FFZ, JYM, XYZ, LLW, $B Q, S W X$ and SLC performed the experiments, collected blood samples and contributed reagents, materials and analytic tools. JYM, XYZ, LLW, BQ, SWX and SLC helped to draft the manuscript. All authors read and approved the final manuscript.

\section{Acknowledgments}

This work was supported by the National Basic Research Program of China (973 Program) (2014CB541901 and 2014CB541902), the National Natural Science Foundation of China (81025016, 81230072, 31370880 and No. 81421001), the State Key Laboratory of Oncogenes and Related Genes (9114-05), the Key Research Program of the Chinese Academy of Sciences (KJZD-EW-L01-3) and the Program of the Shanghai Commission of Science and Technology (12ZR1435900, 12JC1406000 and 12431900703). The funders had no role in study design, data collection and analysis, the decision to publish or the preparation of the manuscript. The authors extend special thanks to Yuting Qin and Ting La of the Chinese Academy of Sciences for providing technological guidance during the course of this study.

\section{Author details}

${ }^{1}$ Shanghai Institute of Rheumatology, Department of Rheumatology, Renji Hospital, School of Medicine, Shanghai Jiao Tong University, Shan Dong Middle Road, Shanghai 200001, People's Republic of China. 'Institute of Health Sciences, Shanghai Jiao Tong University School of Medicine (SJTUSM) and Shanghai Institutes for Biological Sciences (SIBS), Chinese Academy of Sciences (CAS), Yue Yang Road, Shanghai 200031, People's Republic of China. ${ }^{3}$ Division of Rheumatology and the Center for Autoimmune Genomics and Etiology (CAGE), Cincinnati Children's Hospital Medical Center, 3333 Burnet Avenue, Cincinnati, $\mathrm{OH}$ 45229, USA

\section{Received: 29 November 2014 Accepted: 20 April 2015} Published online: 21 May 2015

\section{References}

1. Rahman A, Isenberg DA. Systemic lupus erythematosus. N Engl J Med. 2008;358:929-39.

2. Baccala R, Hoebe K, Kono DH, Beutler B, Theofilopoulos AN. TLR-dependent and TLR-independent pathways of type I interferon induction in systemic autoimmunity. Nat Med. 2007;13:543-51.

3. Marshak-Rothstein A. Toll-like receptors in systemic autoimmune disease. Nat Rev Immunol. 2006:6:823-35.

4. Wapinski O, Chang HY. Long noncoding RNAs and human disease. Trends Cell Biol. 2011;21:354-61. A published erratum appears in Trends Cell Biol. $2011 ; 21: 561$
5. Shen N, Liang D, Tang Y, de Vries N, Tak PP. MicroRNAs-novel regulators of systemic lupus erythematosus pathogenesis. Nat Rev Rheumatol. 2012;8:701-9.

6. Pauley KM, Cha S, Chan EK. MicroRNA in autoimmunity and autoimmune diseases. J Autoimmun. 2009;32:189-94.

7. Chafin CB, Reilly CM. MicroRNAs implicated in the immunopathogenesis of lupus nephritis. Clin Dev Immunol. 2013;2013:430239.

8. Wang $H$, Peng W, Ouyang $X, L i$ W, Dai Y. Circulating microRNAs as candidate biomarkers in patients with systemic lupus erythematosus. Transl Res. 2012;160:198-206.

9. Wang G, Tam LS, Kwan BC, Li EK, Chow KM, Luk CC, et al. Expression of miR-146a and miR-155 in the urinary sediment of systemic lupus erythematosus. Clin Rheumatol. 2012;31:435-40.

10. Tang Y, Luo X, Cui H, Ni X, Yuan M, Guo Y, et al. MicroRNA-146a contributes to abnormal activation of the type I interferon pathway in human lupus by targeting the key signaling proteins. Arthritis Rheum. 2009;60:1065-75.

11. Guttman M, Amit I, Garber M, French C, Lin MF, Feldser D, et al. Chromatin signature reveals over a thousand highly conserved large non-coding RNAs in mammals. Nature. 2009:458:223-7.

12. Zaratiegui M, Irvine DV, Martienssen RA. Noncoding RNAs and gene silencing. Cell. 2007;128:763-76.

13. Ponting $C P$, Oliver PL, Reik W. Evolution and functions of long noncoding RNAs. Cell. 2009;136:629-41.

14. Gibb EA, Brown CJ, Lam WL. The functional role of long non-coding RNA in human carcinomas. Mol Cancer. 2011;10:38.

15. Gupta RA, Shah N, Wang KC, Kim J, Horlings HM, Wong DJ, et al. Long non-coding RNA HOTAIR reprograms chromatin state to promote cancer metastasis. Nature. 2010;464:1071-6.

16. Haywood MEK, Rose SJ, Horswell S, Lees MJ, Fu G, Walport MJ, et al. Overlapping BXSB congenic intervals, in combination with microarray gene expression, reveal novel lupus candidate genes. Genes Immun. 2006;7:250-63.

17. Wang P, Xue Y, Han Y, Lin L, Wu C, Xu S, et al. The STAT3-binding long noncoding RNA Inc-DC controls human dendritic cell differentiation. Science. 2014;344:310-3.

18. Carpenter S, Aiello D, Atianand MK, Ricci EP, Gandhi P, Hall LL, et al. A long noncoding RNA mediates both activation and repression of immune response genes. Science. 2013;341:789-92.

19. Hu G, Tang Q, Sharma S, Yu F, Escobar TM, Muljo SA, et al. Expression and regulation of intergenic long noncoding RNAs during T cell development and differentiation. Nat Immunol. 2013;14:1190-8.

20. Song J, Kim D, Han J, Kim Y, Lee M, Jin EJ. PBMC and exosome-derived Hotair is a critical regulator and potent marker for rheumatoid arthritis. Clin Exp Med. 2015;15:121-6.

21. Stuhlmüller B, Kunisch E, Franz J, Martinez-Gamboa L, Hernandez MM, Pruss A, et al. Detection of oncofetal H19 RNA in rheumatoid arthritis synovial tissue. Am J Pathol. 2003;163:901-11. A published erratum appears in Am J Pathol. 2003:163:2645.

22. Ban Y, Hirano T. Association studies of the SAS-ZFAT, IL-23R, IFIH1 and FOXP3 genes in autoimmune thyroid disease. Expert Rev Endocrinol Metab. 2009; $4: 325-31$

23. Johanneson B, Lima G, von Salomé J, Alarcón-Segovia D, Alarcón-Riquelme ME. the Collaborative Group on the Genetics of SLE, the BIOMED ॥ Collaboration on the Genetics of SLE and Sjögren's Syndrome. A major susceptibility locus for systemic lupus erythematosus maps to chromosome 1q31. Am J Hum Genet. 2002;71:1060-71.

24. Tsao BP. The genetics of human systemic lupus erythematosus. Trends Immunol. 2003;24:595-602

25. Tsao BP. Update on human systemic lupus erythematosus genetics. Curr Opin Rheumatol. 2004;16:513-21.

26. Li Z, Chao TC, Chang KY, Lin N, Patil VS, Shimizu C, et al. The long noncoding RNA THRIL regulates TNFa expression through its interaction with hnRNPL. Proc Natl Acad Sci U S A. 2014;111:1002-7.

27. Linker-Israeli M, Deans R, Wallace D, Prehn J, Ozeri-Chen T, Klinenberg J. Elevated levels of endogenous IL-6 in systemic lupus erythematosus: a putative role in pathogenesis. J Immunol. 1991;147:117-23.

28. Nagafuchi $H$, Suzuki N, Mizushima $Y$, Sakane T. Constitutive expression of IL-6 receptors and their role in the excessive B cell function in patients with systemic lupus erythematosus. J Immunol. 1993;151:6525-34.

29. McCarthy EM, Smith S, Lee RZ, Cunnane G, Doran MF, Donnelly S, et al. The association of cytokines with disease activity and damage scores in systemic lupus erythematosus patients. Rheumatology (Oxford). 2014;53:1586-94. 
30. Tan EM, Cohen AS, Fries JF, Masi AT, McShane DJ, Rothfield NF, et al. The 1982 revised criteria for the classification of systemic lupus erythematosus. Arthritis Rheum. 1982;25:1271-7.

31. Aletaha D, Neogi T, Silman AJ, Funovits J, Felson DT, Bingham 3rd CO, et al. 2010 rheumatoid arthritis classification criteria: an American College of Rheumatology/European League Against Rheumatism collaborative initiative. Arthritis Rheum. 2010;62:2569-81.

32. Gladman DD, Ibañez D, Urowitz MB. Systemic Lupus Erythematosus Disease Activity Index 2000. J Rheumatol. 2002;29:288-91.

33. Gladman D, Ginzler E, Goldsmith C, Fortin P, Liang M, Urowitz M, et al. The development and initial validation of the Systemic Lupus International Collaborating Clinics/American College of Rheumatology damage index for systemic lupus erythematosus. Arthritis Rheum. 1996;39:363-9.

34. Cohen J. Statistical power analysis for the behavioral sciences. 2nd ed. Hillsdale, NJ: Lawrence Erlbaum Associates; 1988.

35. Rapicavoli NA, Qu K, Zhang J, Mikhail M, Laberge RM, Chang HY. A mammalian pseudogene IncRNA at the interface of inflammation and anti-inflammatory therapeutics. Elife. 2013;2:e00762.

36. Lartigue A, Colliou N, Calbo S, François A, Jacquot S, Arnoult C, et al. Critical role of TLR2 and TLR4 in autoantibody production and glomerulonephritis in Ipr mutation-induced mouse lupus. J Immunol. 2009;183:6207-16.

37. Urbonaviciute V, Starke C, Pirschel W, Pohle S, Frey S, Daniel C, et al. Toll-like receptor 2 is required for autoantibody production and development of renal disease in pristane-induced lupus. Arthritis Rheum. 2013;65:1612-23.

38. Rinn JL, Kertesz M, Wang JK, Squazzo SL, Xu X, Brugmann SA, et al. Functional demarcation of active and silent chromatin domains in human HOX loci by noncoding RNAs. Cell. 2007;129:1311-23.

39. Pauli A, Rinn JL, Schier AF. Non-coding RNAs as regulators of embryogenesis. Nat Rev Genet. 2011;12:136-49.

40. Feng $X$, Wu H, Grossman JM, Hanvivadhanakul P, FitzGerald JD, Park GS, et al. Association of increased interferon-inducible gene expression with disease activity and lupus nephritis in patients with systemic lupus erythematosus. Arthritis Rheum. 2006;54:2951-62.

41. Fu Q, Chen X, Cui H, Guo Y, Chen J, Shen N, et al. Association of elevated transcript levels of interferon-inducible chemokines with disease activity and organ damage in systemic lupus erythematosus patients. Arthritis Res Ther. 2008;10:R112.

42. Walsh RJ, Kong SW, Yao Y, Jallal B, Kiener PA, Pinkus JL, et al. Type I interferon-inducible gene expression in blood is present and reflects disease activity in dermatomyositis and polymyositis. Arthritis Rheum. 2007:56:3784-92.

43. van der Pouw Kraan TC, Wijbrandts CA, van Baarsen LG, Voskuyl AE, Rustenburg F, Baggen JM, et al. Rheumatoid arthritis subtypes identified by genomic profiling of peripheral blood cells: assignment of a type I interferon signature in a subpopulation of patients. Ann Rheum Dis. 2007;66:1008-14

44. Higgs BW, Liu Z, White B, Zhu W, White Wl, Morehouse C, et al. Patients with systemic lupus erythematosus, myositis, rheumatoid arthritis and scleroderma share activation of a common type I interferon pathway. Ann Rheum Dis. 2011;70:2029-36.

45. Petri $M$, Singh $S$, Tesfasyone $H$, Dedrick R, Fry $K$, Lal $P$, et al. Longitudinal expression of type I interferon responsive genes in systemic lupus erythematosus. Lupus. 2009;18:980-9.

46. Landolt-Marticorena C, Bonventi G, Lubovich A, Ferguson C, Unnithan T, $\mathrm{Su}$, et al. Lack of association between the interferon-a signature and longitudinal changes in disease activity in systemic lupus erythematosus. Ann Rheum Dis. 2009;68:1440-6.

47. Lee JT. Epigenetic regulation by long noncoding RNAs. Science. 2012;338:1435-9

48. Spizzo R, Almeida MI, Colombatti A, Calin GA. Long non-coding RNAs and cancer: a new frontier of translational research? Oncogene. 2012;31:4577-87.

49. Saad F. UPM3: review of a new molecular diagnostic urine test for prostate cancer. Can J Urol. 2005;12:40-3. discussion 99-100.

50. Kumarswamy R, Bauters C, Volkmann I, Maury F, Fetisch J, Holzmann A, et al. Circulating long noncoding RNA, LIPCAR, predicts survival in patients with heart failure. Circ Res. 2014;114:1569-75.

51. Guennewig B, Cooper AA. The central role of noncoding RNA in the brain. Int Rev Neurobiol. 2014;116:153-94.

52. Van Roosbroeck K, Pollet J, Calin GA. miRNAs and long noncoding RNAs as biomarkers in human diseases. Expert Rev Mol Diagn. 2013;13:183-204

\section{Submit your next manuscript to BioMed Central and take full advantage of:}

- Convenient online submission

- Thorough peer review

- No space constraints or color figure charges

- Immediate publication on acceptance

- Inclusion in PubMed, CAS, Scopus and Google Scholar

- Research which is freely available for redistribution

Submit your manuscript at www.biomedcentral.com/submit 